\title{
The Change in News Video Production Environment by the Introduction of Network Production System
}

\author{
Sung-Hoon Won, Won-Ho Choi, Kim Cheeyong, Member, KIMICS
}

\begin{abstract}
The production environment of fast and accurate news video has been continuously changing together with technology advance. Especially, the editing of the news video advanced focusing on the non-linear system by the introduction of digital technology and the recording of news video advanced to 'tapeless' base. In this new news video production environment, the recorded news videos are transmitted, edited and archived through the NPS (Network Production System), which dramatically improves the efficiency of the news video production. However, there are some issues which need to be resolved in the introduction of NPS. The purpose of this research is to identify the necessity of NPS introduction in the changing news video production environment, explore the issues which should be resolved and suggest the proper way to introduce the NPS to broadcasting companies.
\end{abstract}

Index Terms - NPS, news production system, news video, non-linear editing, network, archiving

\section{INTRODUCTION}

News transmits information on various fields in the society by reporting the facts. News is transmitted by various media such as newspaper, broadcasting and Internet. Among them, the biggest characteristic of broadcasting news is that it transmits news based on video information. The viewers, who are the news consumers, were not present at the site of news; however, they can get the live and realistic news information by way of news video being broadcasted. Therefore, it can be said that the biggest advantage of the broadcasting news is the news video. Since the beginning of broadcasting until today, production system of news video has been continuously advanced together with the advance in technology.

In the beginning stage of news video production, news was photographed on film like the movie process. The news video was produced by developing and editing the news film. Then the electronic technology came and news video production system advanced to U-matic

Manuscript received December 29, 2010; revised January 28, 2011; accepted February 1, 2011.

Sung-hoon Won is with Dept. of Visual Information Technology, Dong-Eui University, Busan, 614-714, Korea(Email : wonsunghoon (a)daum.net)

Won-Ho Choi is with Dept. of Digital Contents, Dongseo University, Busan, 617-716, Korea(Email : choiwh@dongseo.ac.kr)

Corresponding author : Kim Cheeyong is with Dept. of Visual Information Technology, Dong-Eui University, Busan, 614-714, Korea (Email : kimchee@deu.ac.kr) system and Betacam system. The digital technology was applied to the news video production since the late 1990s for better efficiency and it brought a big change in the news video production environment. The digital technology had been applied for the recording session as the Digibeta recording system and it advanced to HD (high definition) video production system. The digital technology, which realized the high video quality and better convenience in recording, also brought dramatic change in the editing, transmitting and storing of the data video. In the analog system, the editing of news video was done by 'linear editing' which is reproducing and editing the recorded video tape through the VCR. However, in the digital system environment, the digitized video data is edited by the computer. In the 'linear editing', the tape should be searched sequentially; therefore, it took lot of time to find the required video for editing. In the 'non-linear editing' by computer, the digitized video can be searched non-sequentially; therefore, it became possible to find and edit the required video much faster than before. However, the digital video recorded on the digital tape requires the 'digitizing' process which is converting the video into digital data. Therefore, there is still a disadvantage that it requires physical time to 'capture' the recorded video by computer. This process of making the video information into data for 'non-linear editing' was the object of improvement in the news video production environment because the news video production requires fast and immediate reporting. The 'tapeless' recording system was developed and was introduced to overcome this shortcoming. The 'tapeless' system does not record the video on the tape. It stores the video in the memory and blue-ray disk. Videos stored as individual files can be immediately edited, which greatly improved the efficiency in the news video production. This 'tapeless' system is leading the advance at the news video production by securing the practical efficiency in the recording of news video and 'non-linear editing'.

The system than handles the video information recording, editing and storing as the digital data later advanced to the NPS. In NPS, user can access to the server having the video information based on 'non-linear editing' system which enables the environment to produce the news video without limitation in time and space. Accordingly, the news video production environment dramatically improved. 
This research explores the change in the news video production environment brought by the NPS, which is based on the 'non-linear editing' system. The issues which should be resolved for the introduction and implementation of NPS will be examined and the solutions to resolve the issues will be suggested.

\section{PRODUCTION OF NEWS VIDEO}

\section{A. The change in the news video production system}

The production of news video has been changing together with the advance in the recording system. In the beginning of television broadcasting, $16 \mathrm{~mm}$ film camera was used for the news video production. It took lot of time in the developing and editing of the film. When the tape-based U-matic system was developed, the chemical developing and physical editing of film became no longer necessary and news video could be produced in the environment run by electronic mechanism. However, since the camera and the VCR were separated in the Umatic system, it caused inconvenience in news-gathering sites. Then the Betacam system was introduced which has the camera and VCR in one body. It greatly improved the efficiency in the recording of news video.

The analog-method news video production system recorded the video on the magnetic tapes. News video was produced by 1:1 'linear editing' and the graphics, characters and BGM works were required at the transmission stage, or, a comprehensive editing stage was separately required.

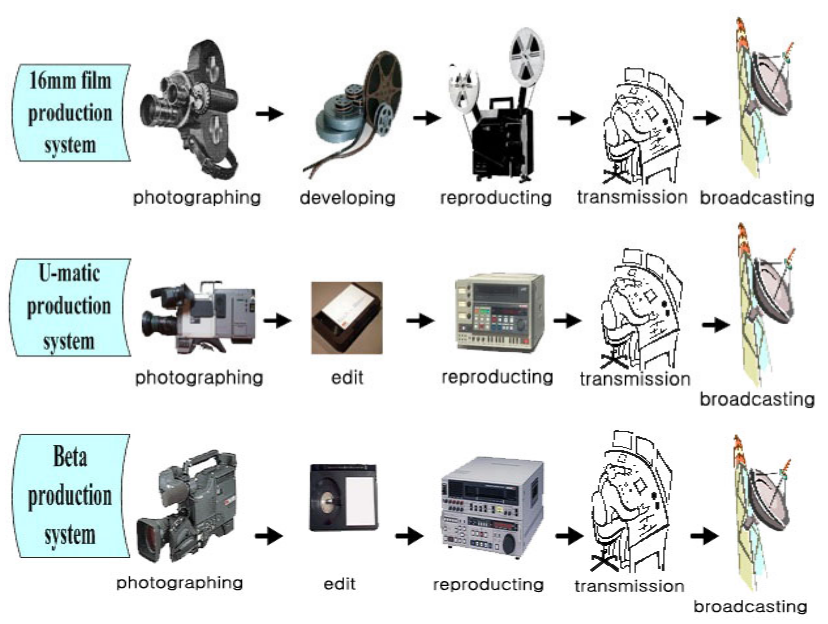

Fig. 1. The analog news video production system

The analog production system such as U-matic or Betacam had limitations because the video quality deteriorated during the repeated processes of editing and copying. The digital processor was introduced to overcome this limitation. The Digibeta system began to be used since the end of 1990s, which was the beginning of the digital video production. It was used in the production site of news video during the transition period between analog and digital. The Digibeta system improved the video quality compared to previous system. The visual deterioration of video quality during the repeated editing and copying was also avoided. The editing was mainly 1:1 method based on the tape and VCR.

The 'non-linear editing' during this 1:1 type editing period was understood as the later-part works to insert the effects of PIP, voice change and mosaic work to protect the right of one's portrait. Therefore, at the time, the 'nonlinear editing' system was generally recognized as a video effect creating system.

The rapid advance of computer system and software development created the practical environment in which the video in digital data form can be edited by the computer. The digital video data stored on the hard disk can be easily searched, edited, deleted or added. Especially, the advantage of 'non-linear editing' received continuous attention in the news video production environment, in which the speed of video editing was critical. This let the utilization of the 'non-linear editing' gradually increase.

Technology advance enabled the environment in which video quality could be further enhanced. HD (high definition) video production system since the early 2000 s brought a turning point in the news video production system by providing with the 2 million pixel resolution, 16:9 picture ratio and $48 \mathrm{KHz}$ high quality audio. The early production environment of news video was developed and distributed based on the tape system. When the broadcasting was in SD (standard definition) grade, the video quality was downgraded to $4: 3$ ratio pictures before broadcasting. Then the broadcasting policy changed in the middle of 2000s. As HD broadcasting became common, the production environment of news video also changed to HD basis.

The HD-base video production environment had been established to make high quality video; however, the tapebased HD production system still needed the 'capturing' to the hard disk. As this became the issue to improve in the news production environment, systems based on memory and disk were developed and distributed. Broadcasting companies began to use the new system in parallel with the existing system.

The biggest reason of broadcasting companies' using various types of news video production systems was that the technology advanced very fast and the standard news video production system had not been established yet. Also, the network-based system to archive the digitized video data had not been established and it caused the mixed use of 'linear editing' system and 'non-linear editing' system in parallel. 


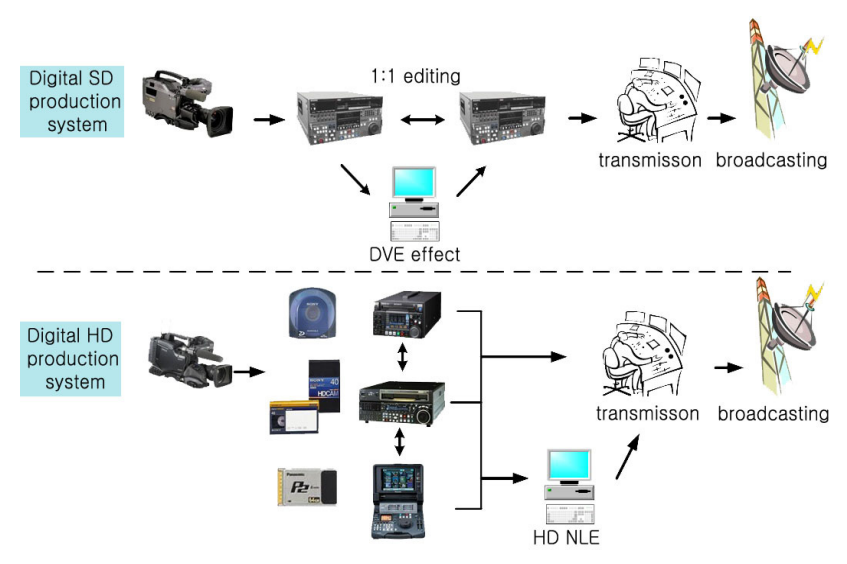

Fig. 2. Digital news video production system

In 2003, KBS Newsroom first used NPS in the news production. Full scale digital news video production system was established when SBS began to use Digital Newsroom and NPS in 2004. In 2005, Jinju MBC first used NPS as the local broadcasting station; however, they established it in SD grade and it had some issues. Seoul MBC introduced NPS in 2007 when they built Ilsan Dream Center. Their NPS was tape-method and filemethod in parallel. In 2009, Seoul MBC Newsroom built its own NPS; however, they also used parallel system in news video production that used tape-method in broadcasting while file-method was used in recording, editing and storing. NPS became a trend which broadcasting companies cannot bypass for the efficiency of news video production. Local broadcasting stations such as Busan MBC are continuously considering the NPS use.

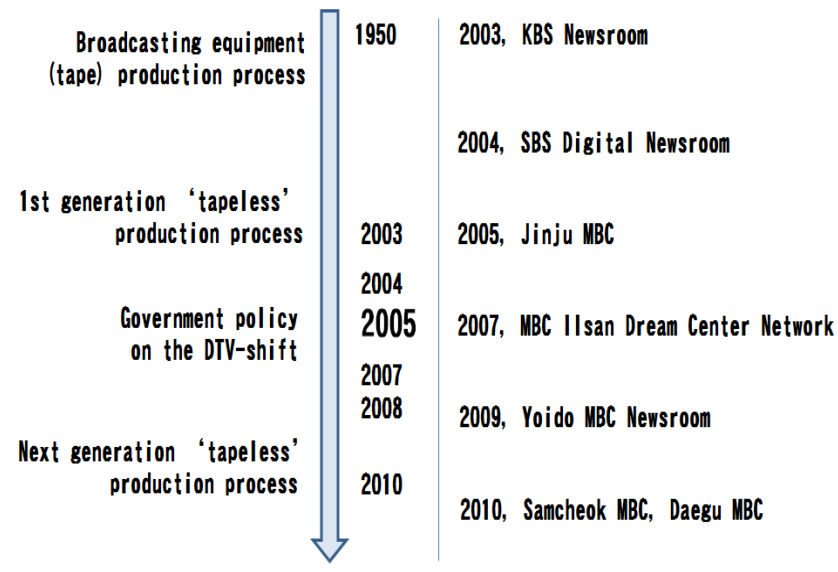

Fig. 3. Present condition of NPS (Samcheok MBC)

The reasons that delay the introduction of NPS are; first, sufficient time and budget for NPS are not secured in advance, and, accordingly, the practical efficiency of 'non-linear editing' system and digital processor are not available yet. The short of awareness on the system operation by broadcasting staff and insufficient leading attitude for a change are other big reasons.

In case of Seoul MBC, all data are stored in files from the Ingesting process after the NPS was introduced and the news video production is done through the network. The 'non-linear editing' has significant meaning in this process. When the previous 'non-linear editing' was a methodology for the news video production, the present 'non-linear editing' in NPS environment, in which all news video production are done through the server and network, became the essential prerequisite. When the previous 'non-linear editing' was an option, the present 'non-linear editing' is an unavoidable method for news production. The news video production system based on network enabled the easy storing/sharing/access of video data. As the efficiency of production enhanced, the paradigm of news video production is making a big turning point with the digital network and the 'non-linear editing' is in the center of the change. NPS and 'nonlinear editing' system can apply video effects and graphics easily together with the editing. Since the news video can be produced anywhere as far as there is a computer linked to the network, the limitation in the time and space can be minimized.

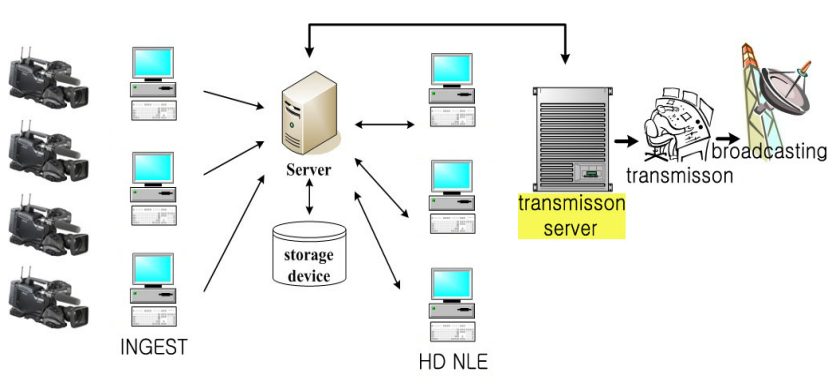

Fig. 4. News video production system based on network

The production environment of news video had been independent and individual but it is now changing to the 'sharing and integrated environment' after the introduction of network system and 'non-linear editing' system. The shift to news video production system based on network brings a revolution which essentially changes the news video production system. And the production infrastructure enabled this change, in which the distribution and utilization of 'non-linear editing' system, 'tapeless', server and network are common.

\section{B. The change in the news video production environment}

The introduction of network and 'non-linear editing' system brings change in the work of news video production staff. The 'tapeless' recording, 'non-linear editing' and network-based NPS can change the newsgathering site as the 'editing and transmission site'. The efficiency of overseas news production can be greatly enhanced by network transmission. In the past, VCR and monitor were essentially required to confirm the recorded 
video and the space for the editing work was also limited. Satellite transmission was the best way from overseas news-gathering site. It required lot of time for recording and editing. When there was a large accident, event or disaster, the OB Van (outdoor broadcasting van) was a must. Now the change in the news video production environment based on the network and 'non-linear editing' is removing the boundaries of time and space for news video production. As the recorded video in digital file at the news video news-gathering site can be edited by the personal notebook and transmitted through the Internet, the production environment of news video dramatically expanded. In addition, the uploaded video data through the network are automatically archived so that they can be utilized anytime, anywhere for news video production. Accordingly, the roles and works of news production staff are also changing.

\section{1) News reporters}

The change in the news video production environment such as network and 'non-linear editing' brought some changes to the work of news reporters. News reporters collect accurate information based on the facts and make it into news articles. Their work area is different from the 'camera reporters'; however, the change in the video production environment brought the change in the newsgathering method of news reporters.

Before, the news reporters prepared the collected articles by hand; however, now they do all the works such as news-gathering, article preparation and article transmission by computer and network. The computer utilization of news reporters provides the news reporters with an opportunity to diversify the methods of newsgathering. News reporters can use the video data while searching the video data stored in the server via computer. They can also prepare articles while seeing the edited news video through the network. They can directly edit the news video if they received training on the 'non-linear editing' software. Therefore, it can be said that the activity area of news reporters is getting bigger while the boundary of news video production becomes ambiguous.

Recently, news reporters actively participate in the 'non-linear editing' training. This reflects their interest in the news video production and the work area expansion of news reporters.

\section{2) Camera reporters}

Camera reporters are the 'video recorders' who record the historical facts through camera at news-gathering sites of accident and events. They provide the viewers with live feeling at site while recording the video and sometimes doing the video editing in addition. In the previous analog system, the news video production was editing the recorded video from site and the articles from news reporters. In this system, it was essential to secure the original video.
However, as the video recording method changed from tape-method to memory or disk methods, an environment in which multiple staff can search and utilize the video has been enabled, once the video would go through the process of 'ingesting' and 'uploading'. Accordingly, the work of the camera reporters also changed. Now they need to directly edit the recorded video and transmit it through network for faster news reporting. The utilization of 'non-linear editing' is again essential in this stage. In other words, only the staff that can do the 'non-linear editing' can do the job. In the news video production system run by network and 'non-linear editing', the camera reporters should have capability to do the editing, transmission and storing of the video, in addition to the recording of video.

\section{3) Editing reporters}

In the analog system, all editing works were done in the editing system based on the tapes and VCR. Editing was possible only after the recording of the camera reporters and it should be done in the editing room. Required data video should be searched in the individual tapes and edited afterwards. It took so much time to find the data video that the staffs said that "if we find the data video, we can say the editing work is completed". Furthermore, if video effects were required for the news video, supports from the 'effect machines' and technical staff were essential. All editing were done in linear method and support of manpower in various fields was required.

As the editing system based on the 'non-linear editing' and network was introduced, the work of editing reporters had massive innovation. A single editing reporter can do all the editing works such as video editing, video effects, subtitles and graphic. The efficiency of news video production greatly improved and the number of staff can be reduced a lot. As the news video editing environment which required many staff before has improved, the production of 'special feature program' can be done without the support of comprehensive editing and technical people. The job efficiency of editing reporters is greatly improved as the search of video data became easier in the full scale network environment.

\section{THE EFFECT AND ISSUES IN NPS AND 'NON-LINEAR EDITING'}

\section{A. The introduction effect of NPS and 'non-linear editing'}

The NPS and 'non-linear editing' greatly improved the job efficiency. Many production staffs are no longer required and video transmission became easy. Anybody who can access the network can search the video data. Therefore, the efficiency in the editing and correction of news video greatly improved and the news video desired by the editors can be produced anywhere, anytime. The 
NPS and 'non-linear editing' system is building the future-aiming broadcasting system. The practical usefulness of archiving has been secured through NPS. Accordingly, shared-utilization of existing video data, direct editing at the news-gathering site and remoteediting became possible. In other words, the physical limitations in the news video production have been overcome.

The NPS and 'non-linear editing' system also brought the effect of budget saving. It requires lot of budget in the beginning to build the system; however, in a long term, the cost of archiving and management of video data is saved and new added value can be generated from the viewpoint of utilization. In other words, the contents value of news video and data video can be maximized and external competitiveness is also enhanced through the building of infrastructure.

TABLE I.

\section{EXPECTED EFFECTS OF NPS INTRODUCTION}
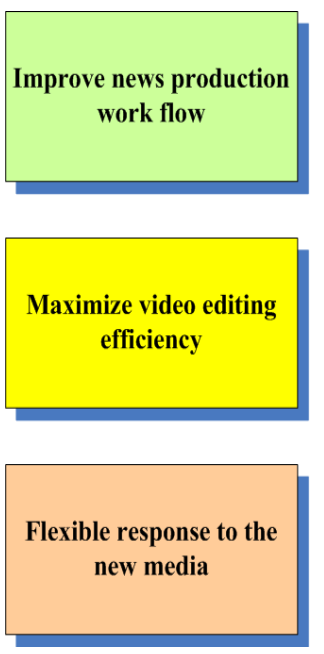

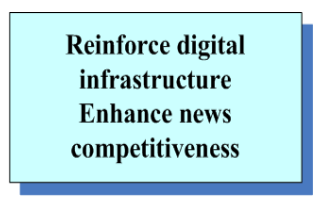

\section{NPS}
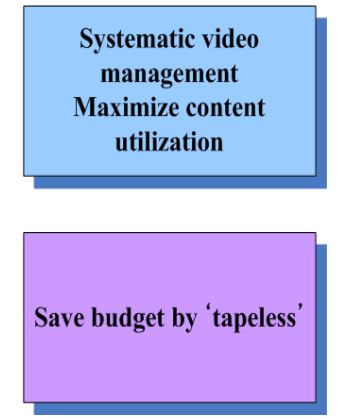

\section{$B$. The issues to be resolved in the NPS introduction}

The NPS provides news video production with excellent efficiency; however, there are realistic issues which need to be resolved.

The first issue is the infrastructure building which requires lot of budget in the beginning. NPS provides with excellent usefulness after it has been built; however, it requires huge budget for the large capacity server, archiving system and network building. Many broadcasting companies cannot afford to make such investment.

The second issue is the decrease in the values of system, manpower and budget because the utilization of existing system will decrease. After shifting to digital system, shifting to HD will continue in Korea. The NPS will decrease the usefulness of existing digital tape method and the editing system based on the HD deck. The usefulness of production manpower already-trained and investment-made would decrease a lot.

Third, even the NPS introduction is necessary from macroscopic viewpoint, the present broadcasting manpower is mixed with people capable of analog broadcasting, digital broadcasting and 'non-linear editing'. The manpower that has knowledge in the NPS operation is not enough. It is not easy to find the new manpower for the news video production and existing news video people are getting older. Sometimes, it is difficult to reach the consensus on the building of NPS.

Fourth issue is that, as the work areas of existing news video production manpower overlap with each other, the work areas of them become ambiguous and there is a concern of possible conflict. This potential can cause the change in the work area by 'open production system' and the reduction of manpower cannot be avoided.

The last issue is how to cope with the possible broadcasting accident during the system stabilization period. Different from analog system, the digital network system like NPS has many elements which can cause the errors and user mistakes. Therefore, the potential for unexpected broadcasting accident will always exist until enough understanding and experience of news video production staffs can be cumulated.

The introduction of NPS is essential to enhance the news video quality, to improve news video production and to catch up with global trend of news video production. The existing news video production staffs also agree on this in principle. However, there are still issues as above which need to be resolved.

\section{How to introduction the NPS}

Now the NPS is a trend which we cannot avoid in the building of infrastructure for news video production. However, since there are issues which need to be resolved for full scale introduction, it is suggested to introduce NPS with step by step approach.

The first step of NPS introduction will be the introduction of 'non-linear editing'. The 'non-linear editing' is already commonly used in terrestrial broadcasting companies and cable television companies.

The second step will be the building of network following the introduction of shared storage. The production system can be built through the 'non-linear editing' system and shared-storage.

The third step will be the making of broadcasting system as a network. All video data should be 'tapeless' in production and transmission after 'ingesting'.

The fourth and last step will be the building and operation of integrated NPS. The news video production and broadcasting viewpoint production will be integrated and it will be operated through the server which will replace the VCR.

Several local broadcasting companies including Busan MBC completed up to Step 2 above. They have target to finish the analog broadcasting and build complete network by 2013 . 
This stepwise introduction of NPS will be able to resolve the realistic issues by way of manpower training, stable infrastructure building and distribution of the budget. Of course, an organized and continuous operation of training program would be required. It is necessary to cope with the changing environment by making the potential factors as manuals. The stepwise introduction of NPS will also help in this area. It can secure the time for sufficient training and companies can refer to cases of other broadcasting companies to cope with their own issue in advance. In addition, the stepwise change of manpower system will mitigate the impact following the environment change.

As the introduction of NPS would be delayed, the potential issues can be minimized; however, the relative competitiveness of broadcasting will also weaken. Therefore, it is required to control the timing of introduction and actively cope with the adaptation of broadcasting company staff.

\section{CONCLUSION}

The video environment is quickly changing after the introduction of digital technology. In the news video production which requires speed, this change works more sensitively. However, the system change should secure both efficiency and stability in the production and various aspects including the budget should be considered.

The introduction of NPS is unavoidable trend now in the news video production. The terrestrial broadcasting companies in Greater Seoul Metropolitan Area already completed the introduction of NPS. Local broadcasting companies are also plan ning to introduce NPS. All these make the environment in which the efficiency of news video production is enhanced and produced contents can be utilized in stable way by archiving the produced news video.

The environment of broadcasting is quickly changing. Multi-media and multi-channels environment already arrived, in which only superior contents can have the relative superiority. The superior positions of broadcasting companies have been lost and they should overcome the existing limitation in news video production in the paradigm of hot competition. NPS is the alternative to cope with this environment and situation.

From macroscopic viewpoint, NPS can enhance efficiency, save production cost and minimize the operation manpower; however, there are many issues to be resolved. They are huge budget, obsoleteness of existing system, re-training for NPS operation, experienced manpower availability, potential conflicts caused by the change in work areas and the stabilization of system operation. However, these issues should be resolved to build the future-aiming news video production and it is suggested that the NPS would be introduced with stepwise approach. The stepwise approach will distribute the budget and mitigate the impact caused by manpower and system stabilization. Of course, continuous re-training and making manuals on the potential issues through investigation and research is essential to cope with the potential issues.

NPS with the Seoul area terrestrial broadcasting companies are completed and they are in the stabilization stage. However, for the local broadcasting companies, who are planning the NPS introduction in the future, the stepwise approach will be a valid alternative. However, if the introduction timing would be excessively postponed in the stepwise introduction to minimize the potential issues danger and mitigate the impact, the relative competitiveness will weaken. All broadcasting staffs should actively cope with future-aiming news video production and realize the advance in the news video production through NPS.

\section{REFERENCES}

[1] Ahn Tae-yeong, "Research and analysis on the video editing system utilization based on HD in HD broadcasting", Graduate School of Comprehensive Art, Kukmin University, 2007.

[2] Hong Chang-yong, "Research on the broadcasting video digital archive system following the digitization of terrestrial TV broadcasting", Graduate School of Media, Seogang University, 2003.

[3] Jang Seok-ho, TV news report editing, Cha-seung Publishing, 2001.

[4] Jeong Jong-won, Future prospect and development of video editing system Vol.9, Dongseo Media, 2005.

[5] Jeong Yong-hwa, "Research on the news production environment change following the news digital system introduction to the terrestrial TV broadcasting companies", Graduate School of Policy and Science, Korea University of Foreign Studies, 2005.

[6] Kim Cheol-hyeon, Baek June-gi, "Research on the threedimensional video production flow based on the non-linear editing", Journal of The Korean Society of Broadcasting Engineers, Vol. 15-3, 2010.

[7] Lee Seon-myeong, "Research on the organization change and performance of broadcasting companies after the shifting-to-digital of broadcasting news production process", Graduate School of Media, Seogang University, 2004.

[8] Samcheok MBC, "2010 new media solution seminar", New Media Solution Seminar, 2010.

[9] Seol Jin-a, Han Seong-soo, Broadcasting Production Technique, Korean National Open University Publishing, 2004.

[10] Yang Yong-cheol, Site manual on news video for HD news production, Korean Broadcasting and Camera Reporters Association, 2009.

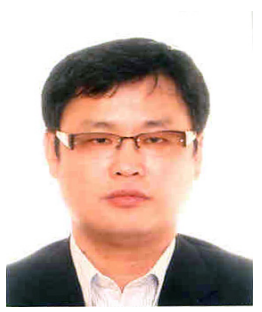

Sung-Hoon Won born in 1973. He is a president of One Media, Pusan, Korea. He received Media Arts \& Science in degree Korea National Open University, Korea in 2009. He is under the master's course in Dept. of Visual Information Technology, Graduate School of Visual Information, Dong-Eui Univ, Pusan, Korea. His research interests are non-linear editing and Broadcasting Image.

Email: wonsunghoon@daum.net 


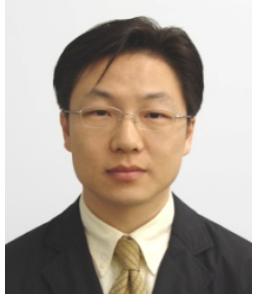

Won-Ho Choi Born in 1972. He is a professor of Dept. Division of Digital Contents at Dongseo University. He is currently a $\mathrm{Ph} \mathrm{D}$. candidate Dept. Interdisciplinary Program of Art-Culture at Pusan National University. His research interests are Image, Visual Art, Unconsciousness and Steroscopic Image.

Email: choiwh@dongseo.ac.kr

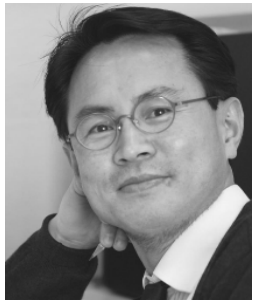

Kim Cheeyong Born in 1964, Pohang, Korea $\mathrm{He}$ received the Ph.D. degree in Computational Physics from Inje University, Republic of Korea, in 2000. He worked at Inje University, Busan College from 1991 2002 and at Dongseo University from 2003-2005. In 2006, he joined the Dong-Eui University, where he is an associate professor. His research interests are in 3D Animation, Multimedia design and VR, Web 3D Contents Design, Fractal \& Chaos design, Conceptual Design. Email: kimchee@deu.ac.kr 